pregnant - based on their sexual activity, nonuse of contraception or use of specific contraception methods, and method-specific contraceptive failure rates. The PRI was compared with current pregnancy, based on self-report and urine testing. Child marriage was measured as ever-married before age 18. Statistical evidence for change over time was assessed using regression analyses with robust variance estimation. The sample included 15,606 women-rounds of observation.

Results School enrollment rose from 26\% in 1994 to $61 \%$ in 2018 ( $\mathrm{p}<0.001)$, coinciding with a national policy of universal primary education instituted in 1997 and considerable increases in household SES. Rates of orphanhood declined from $52 \%$ in 2004 to $23 \%$ to 2018 ( $\mathrm{p}<0.001$ ), corresponding to availability in antiretroviral therapy from 2004. Child marriage among women 15-19 years declined from 33\% to $4 \%$ $(\mathrm{p}<0.001)$. Current pregnancy declined by $65 \%$; a parallel $58 \%$ decline in the average PRI score reflects a decline in sexual experience $(67 \%$ to $40 \%)$ and increases in current contraception use $(29 \%$ to $42 \%$, all trends $\mathrm{p}<0.001)$. Adjusted for age and survey rounds, school enrollees compared to nonenrollees reported less sexual experience $(43 \%$ vs. $79 \%$, $\mathrm{p}<0.001)$, greater use of condoms $(55 \%$ v $20 \%, \mathrm{p}<0.001)$ and greater use of any contraceptive method $(61 \%$ v $39 \%$, $\mathrm{p}<0.001)$.

Conclusions Adolescent pregnancy and child marriage declined from 1994 to 2018 as enrollment in school and socioeconomic status increased and HIV-related orphanhood declined. Social determinants can have an enormous influence on adolescent health and social transitions.

\section{P7 TRENDS, DETERMINANTS AND INEQUALITIES IN ADOLESCENT MOTHERHOOD IN 74 LOW AND MIDDLE- INCOME COUNTRIES: A POPULATION-BASED STUDY}

\begin{abstract}
${ }^{1,2} \mathrm{MM}$ Huda, ${ }^{1,2} \mathrm{M}$ O'Flaherty, ${ }^{3} \mathrm{JE}$ Finlay, ${ }^{1,2} \mathrm{AA}$ Mamun. 'Institute for Social Science Research (ISSR), The University of Queensland, Brisbane, Queensland, Australia; ${ }^{2}$ Life Course Centre, ISSR, The University of Queensland, Brisbane, Queensland 4068, Australia; ${ }^{3}$ Department of Global Health and Population, Harvard T.H. Chan School of Public Health, Boston, USA
\end{abstract}

10.1136/bmjpo-2019-RCPCH-SAHM.15

Aims Reducing adolescent motherhood is an important indicator of several global health-related goals. Assessing the epidemiological burden of adolescent motherhood is important in supporting prevention initiatives to achieve these goals. Thus, the purpose of this study is to examine the trends, inequalities and determinants of adolescent motherhood in low- and middle-income countries (LMICs).

Methods We analysed 238-nationally representative demographic health surveys conducted between1990-2016 in 74 LMICs. The annual weighted prevalence of adolescent motherhood was estimated, and their trend was examined using time-series method. We estimated and compared the average annual rate of change (AARC) in adolescent motherhood. Inequalities in adolescent motherhood along different sociodemographic characteristics were described using the normalized concentration index $(\mathrm{C})$ proposed by Wagstaff. A generalized estimating equation model was used to identify determinants for adolescent motherhood.

Results In total, 704,077 adolescent girls (15-19 years) were included in this study. The average weighted prevalence of adolescent motherhood was $19.46 \%$ (95\%CI, $18.16 \%$ $20.75 \%$ ) during 1990-2016. The prevalence varied from $7.20 \%$ to $24.90 \%$ across different regions, with the highest prevalence in Sub-Saharan Africa. Adolescent motherhood declined (AARC $=-0.80 \%)$ in LMICs with some variations across regions and countries. The highest decline was observed in South \& Southeast Asia (AARC $=-1.79 \%$ ) whereas no reduction was observed in the Latin \& Caribbean region. Further, $28.10 \%(16 / 57)$ of the studied countries exhibited increasing in adolescent motherhood. Significant inequalities in adolescent motherhood were observed by wealth quintile $(\mathrm{C}=-0.249)$, level of education $(C=-0.215)$, area of residence $(C=-0.138)$, and exposure to media $(C=-0.069)$. Pooled adjusted model showed that wealth quintile, employment status, media exposure, early marriage, knowledge about ovulation, partner's greater age difference, and partner's desire for more children are significant determinants for adolescent motherhood.

Conclusion Overall reductions in the prevalence of adolescent motherhood were observed in LMICs; however, inequalities in the prevalence persist. There was no progress in reducing the prevalence in some high burden countries. Early marriage, partner's age difference, and their desire for more children are consistently identified as determinants for adolescent motherhood in most of the region. International policymakers could be beneficial from these findings in designing interventions to prevent adolescent motherhood.

\section{P8 ADOLESCENT PSYCHOSOCIAL HISTORY USING HEADSS IN A TERTIARY PAEDIATRIC EMERGENCY DEPARTMENT}

K Sullivan*, H Samarendra, K Malbon, D Orteu. Women's and Children's Health, Imperial NHS Healthcare Trust, London, UK

\subsection{6/bmjpo-2019-RCPCH-SAHM.16}

Aims To describe current practices and referral outcomes using HEADSS psychosocial screening for adolescents presenting to the Emergency Department at a tertiary metropolitan referral hospital.

Methods Hospital records of patients aged 13 to 20 attending the emergency department were reviewed over a 4week period. Basic patient demographics, presenting complaint and the role of the health professional documenting the HEADDSS assessment was noted. Records were assessed for documentation of psychosocial history items in accordance with the HEADSSS psychosocial screening tool. The number and type of referrals resulting from HEADSS screening was recorded. Data was analysed using basic statistical methods.

Results 363 adolescents aged 13 to 20 years attended the Emergency Department during the study period. Documentation of persons present during HEADSS screening was often incomplete However, only $7 \%$ of adolescents were seen alone. HEADSS screening was largely completed by doctors, with just $17 \%$ of performed by nurses. Overall, HEADSS screening rates were poor. $43 \%$ of patients were not asked about any aspect of HEADSS psychosocial screening. $60 \%$ of adolescents were asked about at least one category, but less than $2 \%$ had a complete HEADSS screening performed. Home, education and substance use were the most frequently asked about categories. Activities, mental health and sexuality were asked about less commonly, while 
eating and body image were rarely discussed. 118 referrals were made as a result of HEADSS assessments; which constituted $54 \%$ of all encounters in which at least 1 category was screened. Referrals were limited in their scope, the majority being made to Social Services and safeguarding; smaller numbers were made to CAMHS, Red Thread and local Sexual Health services.

Conclusion The use of HEADSS psychosocial screening, which was largely performed by doctors, was inadequate amongst the 13 to 20 year age group. The high rate of referral when HEADSS screening was performed suggests it is of value. This data also suggests a need for multidisciplinary education around HEADSS screening, and exploration of referral pathways to other services that address the needs of young people presenting to the Emergency Department.

\section{P9 GETTING IT RIGHT FOR ADOLESCENTS: WHAT DO THEY WANT? PATIENT EXPERIENCE OF A HOSPITAL STAY}

${ }^{1,2} \mathrm{~K}$ Vutipongsatorn*, ${ }^{1,2} \mathrm{~V}$ Ramadoss, ${ }^{2} \mathrm{D}$ Preston, ${ }^{2} \mathrm{~K}$ Malbon. 'Department of Medicine, Imperial College London, London, UK; ${ }^{2}$ Department of Paediatrics, St Mary's Hospital, London, UK

\subsection{6/bmjpo-2019-RCPCH-SAHM.17}

Aims To assess the experiences of adolescent patients in an inpatient setting at a tertiary hospital.

Methods From October 2018 to March 2019, patients aged 13-23 years old were identified using the electronic record system and were approached to complete an anonymous 29question survey. The survey asked participants about their well-being, appropriateness of environment and awareness of adolescence-related services.

Results 30 patients completed the survey. 48\% were under sixteen years old while $41 \%$ were sixteen to twenty-one. Approximately half (47\%) were male. $63 \%$ and $37 \%$ were admitted to the paediatric and adult wards respectively. 90\% felt this was appropriate. Among the 10\% who indicated otherwise, reasons given were 'being frightened' by older patients and 'sharing toilets'. The majority (93\%) felt that they understood their condition. However, 83\% reported that they had a say in management with 53\% feeling that their parents did most of the talking during consultations. The most common topics that participants would like to discuss were education (43\%), mental health (29\%) and weight management (24\%). 28\% suffered from low mood or felt the need to seek mental health support. $41 \%$ did not know where to access support for sexual health and contraception. $32 \%$ had discussions about alcohol, smoking or drugs. Among these, only 38\% were told about options for cessation and further help. Three themes were identified as suggested areas of improvement. 1. Participants would like improved facilities with individual toilets, study rooms and private space for adolescents. 2. More entertainment options such as game consoles and better Internet access were proposed. 3. Patients felt they would benefit from more nurses and a quicker discharge process.

Conclusion Adolescents should be placed in more appropriate environments when admitted to hospital to maximise the comfort of their stay, co-horting patients with those of a similar age and providing young person friendly entertainment and resource. Additionally, professionals should opportunistically provide education about their physical and mental health and emotional wellbeing. Finally, referral pathways to community partners need to be established to aid discharge and provide support once a young person has left the hospital.

\section{P10 FEASIBILITY, ACCEPTABILITY AND EFFECTIVENESS OF YOUNG-PEOPLE SPECIFIC, INTEGRATED OUT-OF- HOSPITAL SERVICES: A SYSTEMATIC REVIEW}

${ }^{1,2}$ A Pandey* ${ }^{*}$ R Viner, ${ }^{1}$ A Gireesh. ${ }^{1}$ Population, Policy and Practice Programme, UCL GOS Institute of Child Health, London, UK; ${ }^{2}$ National Guideline Alliance, Royal College of Obstetricians and Gynaecologists, London, UK

\subsection{6/bmjpo-2019-RCPCH-SAHM. 18}

Aims Although there is a broad agreement on the need of integrated care in young people, there has been no systematic effort to evaluate the provision of integrated out of hospital health services for this group. The objective of the review was to examine the effectiveness, feasibility, and acceptability of young-people specific integrated out-of-hospital services.

Methods We systematically searched for relevant peer-reviewed articles published before 2018 using an iterative search strategy: Medline, EMBASE/Ovid, CINAHL Plus and CENTRAL. In addition, a hand search of reference lists and relevant topics from systematic reviews and grey literature were also performed. Data were extracted and synthesised across these three themes: (i) effectiveness, (ii) feasibility and (iii) acceptability of integrated out-of-hospital health services for young people (10-24 years).

Results Following title and abstract screening, 177 full text articles were reviewed and 31 studies meeting the inclusion criteria were included and summarized in a narrative review. Services offered varied across different studies. Fourteen services focused on mental health services. Other services either had a holistic focus based within primary care, provided interdisciplinary care, HIV and pregnancy prevention services or reports were unclear in their descriptions of the service focus. The most common intervention described was individual counselling. Services were generally acceptable and effective, though a small number also experienced no change or a decline in their condition. Common features of effective and acceptable programs were being youth-friendly (staff and environment) and welcoming, being staffed by young people, maintaining confidentiality and privacy, wide range of integrated services available in one place, convenient location, community-based outreach services, disease-oriented care facilitation, and integration within existing services, nurse/peer-led care, consistent service and collaborative governance. However inconsistency in findings was observed and quality of evidence was weak.

Conclusion There is some preliminary evidence on the effectiveness and acceptability of out-of-hospital integrated youth services, but, limited evidence to support the feasibility. Further research is required to evaluate the strength of such youth-focussed services in order to maximise patient benefit and to inform public policy. Considering the limitations in current evidence base including lack of direct comparison of services; large comparative studies controlling for possible confounders is merited. 